
\title{
25 Research Soure \\ Loss of FANCD2 and related proteins may predict malignant transformation in oral epithelial dysplasia
}

\author{
Mark Ryan \\ University of Liverpool \\ Juhi Gupta \\ University of Liverpool \\ Asterios Triantafyllou \\ University of Liverpool \\ Janet Risk \\ University of Liverpool \\ Richard Shaw \\ University of Liverpool \\ James Wilson \\ University of Liverpool
}

Michael W Ho ( $\nabla$ m.ho@leeds.ac.uk )

Leeds Teaching Hospitals NHS Trust https://orcid.org/0000-0001-9810-3136

\section{Research article}

Keywords: oral epithelial dysplasia; FANCD2; DNA damage repair; malignant transformation; oral leukoplakia; oral cancer; early detection

Posted Date: March 29th, 2020

DOI: https://doi.org/10.21203/rs.2.17496/v4

License: (c) (1) This work is licensed under a Creative Commons Attribution 4.0 International License.

Read Full License

Version of Record: A version of this preprint was published at Oral Surgery, Oral Medicine, Oral Pathology and Oral Radiology on July 1st, 2021. See the published version at https://doi.org/10.1016/j.00oo.2021.07.001. 


\section{Abstract}

Background: Predicting malignant transformation in oral epithelial dysplasia(OED) is a clinical challenge. The higher rate of malignant transformation in non-smokers supports an endogenous aetiology. Loss of FANCD2 and associated proteins could lead to genomic instability and oncogenesis.

Methods: Longitudinal archival samples from 40 individuals with OED from time of diagnosis to the most recent review in 23 stable OED; or until excision of the SCC in 17 unstable OED undergoing malignant transformation. Histopathological reassessment, immunohistochemistry for FANCD2 and Western blotting for phosphorylation/monubiquitination status of ATR, CHK1, FANCD2 and FANCG were undertaken on each tissue sample.

Results: Decreased expression of FANCD2 was observed in the diagnostic biopsy of OED lesions which later underwent malignant transformation. Combining the FANCD2 expression scores with histological grading more accurately predicted malignant transformation $(p=0.005)$ than histology alone and correctly predicted malignant transformation in 10/17 initial biopsies. Significantly reduced expression of total FANCD2, pFANCD2, pATR, pCHK-1 and pFANCG were observed in unstable OED.

Discussion: There is good evidence that defects in the DNA damage sensing-signalling-repair cascade are associated with malignant transformation in OED. Loss of post-translational modification in FANCD2 and related proteins, was more predictive of malignant transformation when compared to clinicopathological parameters.

\section{Background}

In the management of oral epithelial dysplasia (OED), histopathological grading and clinical determinants of malignant transformation to oral squamous cell carcinoma (OSCC) have been the primary influence in the treatment approach adopted (1-3), despite the numerous studies identifying putative molecular and other predictors of malignant change (4-6). Most studies aimed at identifying a molecular or pathological marker of malignant change have failed to undertake correlation with longitudinal clinical outcomes, so their translational value has been diminished leading to the lack of clinical application (7-9). Furthermore, they have mostly not been formally validated in independent series - this approach is difficult as the event rate of malignant transformation is low, and studies are therefore prolonged (3).. The clinical outcomes of a cohort of patients managed in the Liverpool Multidisciplinary Oral Dysplasia Clinic, identified non-smoking status and the non-homogenous appearance of OED as the strongest independent predictors of malignant transformation (HR 5.9 and 2.3 respectively) (1). The estimated malignant transformation rate in this study was $22 \%$ over 5 years. The more aggressive behaviour of lesions observed in non-smoking patients (or light smokers) with OED supports an endogenous aetiology: while this might seem counter-intuitive, this trend has been seen in other models of carcinogenesis $(10,11)$. 
The incidence of head and neck squamous cell carcinoma (HNSCC) in patients with the cancer prone syndrome, Fanconi Anaemia, is 1400 times greater than that of the general population $(12,13)$ and occurs earlier in life. The Fanconi Anaemia pathway (FAP) removes interstrand crosslinks (ICL) lesions and facilitates homologous recombination repair of DNA double-strand breaks and is an integral component of the DNA damage repair mechanism which maintains genomic stability in healthy individuals (14-16). The FAP consists of 22 proteins (FANCC - FANCW) and has 3 main components (17) (Figure 1.1): (i) The FANCM-MHF1-MHF2 complex senses ICLs and localises to the DNA, acting as a recruitment site for the core complex through FANCM-FANCF interactions. The main role of the core complex is to facilitate ubiquitination of (ii) FANCD2 and FANCI protein dimers. The core complex mediates FANCD2/FANCI monoubiquitination, at residues K561 and K532 through FANCL E3 ubiquitin ligase activity, which then activates (iii) downstream effector proteins which include nucleases, translesion polyermases, homologous recombination proteins, and de-ubiquitinases to complete the DNA repair process and to attenuate FAP signalling. The detection of prognostic markers of DNA damage such as single-stranded DNA and replication stress, leads to the ataxia telangiectasia rad-3 (ATR) and checkpoint 1 (CHK1) kinases mediated cell cycle arrest in $\mathrm{G} 2$ or S phase, and activation of key components of the FA pathway via phosphorylation at several functional residues, including; FANCA at S1449, FANCM at S1045, FANCD2 at S717, S222, S331 and T691, FANCG at S387, S383 and S7, and FANCE at S374 and T346. (18-21).

Damaged and left unrepaired, ICL lesions which progress through the cell cycle, can lead to stalling of replication forks and the eventual formation of double-strand breaks, that results in genomic instability. (22).

We hypothesise that in transforming OED (particularly in non-smokers), an aberration in the DNA damage sensing, signalling or repair pathways leads to accumulation of DNA mutations and malignancy.. In OED, the potential exists for identification of these aberrations in these lesions with potential for malignant transformation. The objective of this study was to investigate the status of FANCD2 and related proteins (ATR, Chk1 and FANCG) in the DNA damage repair pathway of OED in patients who presented to the Liverpool Multidisciplinary Oral Dysplasia clinic, specifically to elucidate if this information could be obtained from the initial diagnostic biopsy as a predictive tool which might have been utilised to influence clinical management, and to correlate these findings to clinicopathologic characteristics.

\section{Methods}

\section{Patients}

Forty patients with OED, were identified from the clinical cohort in Chapter 2 and were included in this study after giving informed consent. All specimens for this study additionally met the additional criteria that the diagnostic FFPE block had not been previously utilised for translational research. The tissue size on the diagnostic biopsy FFPE block allowed for four $0.6 \mathrm{~mm}$ core to be extracted for RNA extraction/Western blotting (23 non-transforming OED - NT and 17 malignant transforming OED). Four 
micrometre sections of the initial diagnostic incisional biopsy FFPE from each patient were stained with haematoxylin and eosin (H\&E) and reported blinded and independently by two oral and maxillofacial pathologists to confirm the presence of OED (Initial attempts to construct a tissue micro-array was unsuccessful as the thickness of the incisional biopsy FFPE specimen was variable and inadequate to allow reproducibility of sections which enabled consistent and meaningful comparison(7)). In this study, the WHO 2017 dysplasia classifications(23) were then grouped as follows: Group 0: severe dysplasia and Group 1: mild or moderate dysplasia

\section{FANCD2 Immunohistochemistry.}

FFPE sections from each clinical timepoint, including the initial diagnostic biopsy, for these 40 patients were stained with anti-FANCD2 antibody (F117: sc-20022, Santa Cruz diluted 1/100) and Biogenex Supersensitive Polymer HRP detection kit (Launch Diagnostics QD430-XAKE) as previously described(11). The expression of FANCD2 was scored blinded by two independent observers (Asterios Triantafyllou and Michael Ho), one being an oral and maxillofacial pathologist. The stained sections were identified by their pathology identification number alone, thus the observers were blinded to the clinical details and outcome of OED i.e. either stable OED or OED which underwent malignant transformation. Consequently, staining was classified by localisation, extent and intensity using a descriptive binary scoring system (Table 1). Any discrepancy in scoring was reviewed jointly and a mutually agreed score determined. Control tissue from normal and OED areas of tissue adjacent to OSCC from a different cohort of anonymised patients $(n=3)$ were stained using the same method.

\section{Western blotting}

Areas with the highest grade of dysplasia in the incisional biopsies were marked on the H\&E stained sections and $0.6 \mathrm{~mm}$ cores were obtained from the corresponding FFPE blocks (minimum of 2 cores from each block). Cores of the same diameter were obtained from anonymised controls as follows: normal tissue from oesophagus $(n=5)$, areas of OSCC from a different cohort $(n=3)$ and tissue with normal histological architecture located adjacent to $\operatorname{OSCC}(n=3)$.

The protein extraction protocol utilised was modified from one previously published (24): FFPE cores were placed in Eppendorf safe-lock tubes (Eppendorf, Hamburg, Germany) and deparaffinised by incubation at room temperature in xylene for $10 \mathrm{~min}$. After each incubation, the tissue was pelleted at 12 $000 \times \mathrm{g}$ for $3 \mathrm{~min}$, and incubation/centrifugation steps were repeated two more times. The deparaffinised tissue pellets were then rehydrated with a graded series of ethanol (100\% for 5 minutes, $90 \%$ for 5 minutes, $70 \%$ for 5 minutes and a further 5 minutes in 70\%) and centrifuge at 1300 RPM for 30 secs to remove excess ethanol. Following this, $150 \mu$ l of Laemmli sample buffer (Sigma-Aldrich) were added. All samples were subjected to high-temperature extraction at $100^{\circ} \mathrm{C}$ for $20 \mathrm{~min}$, and then cooled in ice for 5 minutes. Extracts were centrifuged for $30 \mathrm{~min}$ at 13000 RPM and the supernatant collected and stored at $-20^{\circ} \mathrm{C}$ until needed. 
The expression of $\beta$-actin, ATR, pATR (s428), CHK1, p-CHK1 (s317), FANCD2 (non-and mono-ubiquitinated isoforms), pFANCD2 (s331), FANCG and pFANCG (s7) were assessed in a blinded fashion (to clinicopathologic details and outcome: stable OED or OED which underwent malignant transformation) by western blotting using anti- mouse or anti-rabbit fluorescently labelled 680/800 secondary antibodies (1:10 000 dilution) (Invitrogen, Paisley UK) (Table 2) (25), extracted protein was separated on SDSpolyacrylamide (PAGE) gels: samples were prepared for loading, by the addition of $20 \mu \mathrm{L}$ of protein extract to $40 \mu \mathrm{L}$ of Laemmli loading buffer and immediately heated at $100{ }^{\circ} \mathrm{C}$ for 5 minutes, prior to loading. The samples were loaded on to a 15\% SDS-PAGE gel which were ran overnight at constant $50 \mathrm{~V}$. The protein was then transferred to a nitrocellulose membrane - 4 hours at constant $350 \mathrm{~mA}$ (26). This was blocked in LI-COR buffer for 1 hour at room temperature prior to the addition of specific antibodies at the specified dilutions.

An Odyssey Infrared Imaging System was used to visualise band intensity (Li-Cor Biosciences, Cambridge, UK). Densitometry values of band intensities were measured and calculated using ImageJ®. For each protein, these values were normalised against the oesophageal normal tissue scores (positive FANCD2 expression (https://www.proteinatlas.org/ENSG00000144554-FANCD2/tissue), anonymised normal tissue readily available), then ranked and divided into tertiles representing low expression, moderate expression and high expression. The normal oesophageal control values always lay within the central tertile.

\section{Data/Statistical Analysis}

Mann Whitney and Fisher exact tests were utilised to compare FANCD2 immunohistochemistry scores between samples from NT and T OED lesions. The Fisher-Freeman-Halton exact test was used as a measure of significant difference and comparison between the densitometry ratios of NT and T OED samples. Statistical analyses were conducted, using the statistical software (IBM SPSS Statistics for Windows, Version 22.0). A p value of less than 0.05 was considered significant.

\section{Results}

\section{Patient outcomes}

The demographic and clinicopathologic details of the forty patients included in this study are summarised in Table 3.

As previously published(1), the malignant transforming $(T)$ group had a higher proportion of non-smokers $(p=0.05)$, lateral tongue lesions $(p=0.01)$ and non-homogenous OED $(p=0.001)$ when compared with the non-transforming (NT) OED group. The median follow-up period was 5.3 years (range 0 - 21 years) with a median of 3.4 years (range 0 - 14 years) for the NT group and 6.9 (range 1 - 21 years) for the T group. The median time to malignant transformation was 3.4 years (range $0-7.6$ years). The dysplasia binary score at first diagnostic biopsy was not significantly different between the groups, with 5/17 (29\%) 
transformers classified as high risk compared with $3 / 23(13 \%)$ non-transformers $(P=0.189)$. These 3 patients were progression-free for 18, 52 and 107 months, respectively, following diagnosis. The median time to malignant transformation for T OED in patients with the higher risk binary score at diagnosis was 14.8 months when compared with T OED with a low risk binary score, 44.7 months $(p=0.1)$.

\section{FANCD2 immunohistochemistry.}

Nuclear and cytoplasmic staining for FANCD2 was absent in morphologically normal oral epithelium (Figure 1A), however, FANCD2 staining increased in intensity in non-invasive dysplastic epithelium and was then lost again in micro-invasive OSCC (Figure 1B). Expression of FANCD2 in dysplastic epithelia was not observed to the degree/pattern of inflammation in the underlying stroma (Figs 1, 2).

A decrease in the intensity of both nuclear and cytoplasmic staining were observed in the first diagnostic biopsy of OED lesions that were destined to undergo malignant transformation when compared with nontransforming OED, although the distribution of staining was not statistically different (the quantitative, rather than qualitative, element of FANCD2 staining was predictive of malignant transformation) (Figure 2; Table 3).

Therefore, only intensity scores were incorporated into a final composite FANCD2-OED score as below. In OSCC sections from transformed OED lesions, both intensity and distribution of FANCD2 staining were reduced compared with the first diagnostic biopsy from the same patient (Table 4).

Combining the FANCD2 nuclear and cytoplasmic intensity scores with the histological grading of OED (mild/moderate dysplasia assigned score of 1 and severe dysplasia/carcinoma-in-situ/SCC assigned score 0 ) produced a score that more accurately predicted transformation $(\mathrm{p}=0.005)$ (Table 5), with a cut off score of 1 or less being significantly associated with a higher risk of malignant transformation in OED $(p=0.001)$. This FANCD2-OED Risk Score in all the retrieved OSCC archival tissue from OED which underwent malignant transformation was $\leq 1$ ( $n=15 ; 2$ archival OSCC specimen not retrievable).

Of the 2 NT OED lesions with low ( $\leq 1)$ FANCD2-OED Risk scores, one patient presented with severe dysplasia which was excised shortly after presentation and has now been progression free for 52 months and one presented with mild dysplasia that has been progression-free for 19 months. Eighty-three percent $(10 / 12)$ of patients with FANCD2-OED Risk score $\leq 1$ in the OED cohort reported went on to develop OSCC compared with $25 \%(7 / 28)$ in patients with a score of $\geq 2$. The diagnostic biopsies for the 7 transforming lesions with high $(\geq 2)$ FANCD2-OED Risk scores were obtained a median of 5.8 years (range 0.3-7.0 years) prior to transformation, while the biopsies from the 10 transforming lesions with low $(\leq 1)$ scores were taken a median of 2.4 years (range 0.2-5.3 years) prior to transformation. In all 15 of the available oral squamous cell carcinomas which had arisen from OED, the expression of FANCD2, and the concomitant FANCD2-OED score, was low (Table 5). The FANCD2-OED scores were not significantly different when the following parameters were assessed: age, gender, site of lesions, appearance of lesion, smoking or alcohol history and time to transformation, but as expected, were associated with grade of 
dysplasia $(P<0.0005)$. In total, $1 / 3$ 'false positive' and 5/12 'false negative' dysplasia grade scores were correctly predicted by the FANCD2-OED score.

\section{FANCD2 and associated protein expression}

No significant differences in the normalised expression of $\beta$-actin, ATR, CHK1 and FANCG were observed between T and NT OED lesions (Table 6; Figure 3). As the biopsy specimens in OED were much smaller in comparison with those obtained in established OSCC, the Western blots were performed on a single occasion due to the limited quantity of available protein.

However, significantly reduced expression of FANCD2 (non-ubiquitinated and mono-ubiquitinated forms), pFANCD2, pATR, pCHK-1 and pFANCG were observed in OED prior to malignant transformation with the FANCD2, pFANCD2 and pFANCG observations mirrored in the control tumour tissue. These parameters did not correlate with any clinicopathological features such as site or smoking history. The median ratio of mono-ubiquitinated: non-ubiquitinated forms of FANCD2 (an indication of activation of the FA pathway) were 0.9 and 0 for NT and T OED, respectively $(p<0.001)$.

\section{Discussion}

The results and observations of this study have evidenced support to the notion that loss of FANCD2 and associated protein (in the DNA damage repair pathway) are associated with malignant transformation in OED. The lower, statistically significant, expression of post-translationally modified proteins in T OED when compared to NT OED is indicative of inactivation of the pathways and was a more accurate predictor of malignant transformation than clinical parameters such as smoking history and site. Furthermore, immunohistochemical staining for FANCD2 coupled with a binary dysplasia score could correctly predict malignant transformation in 10/17 initial biopsy samples obtained prior to transformation, with a 'false positive' rate of 2/23 non-transforming OED biopsies. This performed better than dysplasia grading alone, either as a binary score or the current WHO classification. The significantly different rates of malignant transformation in between patients with low FANCD2 (83\%) expression and higher FANCD2 (25\%) expression further validate its potential utility in the clinical setting in determination of the risk of carcinogenesis. The data of Rudland et al (11) indicates that cytoplasmic as well as nuclear staining may be prognostic, but their complicated system for scoring extent and intensity of staining was not appropriate for our samples which were generally small in nature. There could be scope for future validation studies to utilise resection specimens if necessary, however there is generally some degree of heterogeneity in tissue architecture/grade of dysplasia and interpretation of these findings require further thought and definition of the scoring criteria utilised. Both CHK1 and CHK2 phosphorylate the FANCD2 protein at different sites, but only phosphorylation of the $\$ 331$ residue activates the protein complex. Upstream, the ATR pathway is of interest because when CHK1 is phosphorylated, it in turn activates FANCD2 by phosphorylation. 
The loss of FANCD2 expression has to be viewed within the context of the presence of OED or OSCC, hence the importance of including the grade of OED (or diagnosis of OSCC) into the FANCD2-OED Risk Score (Table 6.5). In several anonymised normal oesophageal control samples(Figure 6.3) the expression of FANCD2 was not observed similar to the normal oral epithelium (obtained from the margins of resected OSCC/OED specimen) (Figure 6.1A). This suggests that in the absence of OED or OSCC, the lack of FANCD2 expression would indicate that the Fanconi Anaemia Pathway has not been activated. The difference in these normal controls with transforming OED is that quite consistently the post-translational modifications e.g. pATR, pChk1, pFANCD2(s331) and pFANCG(s7) have all been preserved in the control specimen. These warrants further investigation in order to explain the mechanism of the Fanconi Anaemia Pathway in normal and diseased tissue within the context of the process of carcinogenesis.

In this analysis, moderate dysplasia was classified together with mild dysplasia as 'low risk' while severe dysplasia was classified as 'high risk'. Other researchers have classified moderate dysplasia as 'high risk', but recent discussion in the literature suggests that both suggested binary classifications are simplistic $(33,34)$. Alternative suggestions for binary classification of oral dysplastic lesions rely heavily on pathological interpretation, which could be prone to intra and inter-observer reliability problems $(35,36)$. It is acknowledged, therefore, that the proposed histopathology/IHC classification in its current format will have skewed the moderately dysplastic lesions in this study towards higher FANCD2-OED scores and is thus prone to false negatives, although it still performed better than histopathology alone. Its strength may be in identifying lesions which will NOT transform, but development into a routine, clinical test requires a more robust definition of reduced immunostaining utilising data from a larger number of transforming and non-transforming lesions. This study is best categorised as proof-of-concept as the design and the cohort are not adequate for a robust prognostic biomarker study. One of our observations was that lichenoid-type inflammatory reactions were seen in the lamina propria of some OED and various carcinomas. These are regarded as secondary and/or host response(s) to changed antigenic profile of dysplastic or tumoral epithelium, and not specifically related to the condition oral lichen planus.

When the NT and T groups were compared, there were significant differences in site, smoking aetiology and appearance between the two cohorts. These are such strong predictors of transformation in our modest cohort that it was impossible to adequately match the two groups. The validity of diagnosis of the grade of OED from an incisional biopsy could potentially be questioned as the issue of heterogeneity of OED especially in a large lesion is a valid consideration. In the Liverpool Oral Dysplasia MDT clinic, the biopsy of OED lesions is carried out by senior surgical members of the team where the area of most clinical concern is sampled. Experience in our practice would suggest that concordance of initial biopsy and definitive histopathology diagnoses were very high and had not adversely impacted on patient outcomes. This was demonstrated in the reported cohort of patients where patients who have their OED excised did not have their grade of OED upgraded but the more often scenario was that the area with the most severe grade of OED has been completely excised by the incisional biopsy. Therefore, the findings observed for FANCD2 immunohistochemistry and Western blotting were valid from the statistical and clinical perspectives. 
Six of the twenty-three NT lesions (that we would have expected to transform) were totally excised therefore some of the differences observed between NT and T groups might potentially relate to the method of treatment rather than the inherent cancer risk of the sample (Table 6.3) This could be a reflection that there is such strong prescription bias on grade and/or clinical features of lesions that it would be impossible to adequately match the cohorts.

The median follow-up period was less in the NT vs the T group [3.4 years (range 0 - 14 years) vs 6.9 (range $1-21$ years)], and this may partially explain the observation of NT OED lesions with low $(\leq 1)$ FANCD2-OED scores, one of whom had only been followed for 19 months, and T OED lesions with high $(\geq 2)$ FANCD2-OED scores, 6 of which were obtained more than 3 years prior to transformation. These data may give some indication as to the sensitivity of this technique for prediction of malignant transformation prior to the event. For example, of the 6 T OED patients in whom we had non-neoplastic tissue from an intermediate timepoint, we observed that the FANCD2-OED scores decreased in 2 patients (from $\geq 2$ to $\leq 1$ in both cases) and remained static in 4 patients ( $3 / 4$ of whom scored $\leq 1$ at the initial biopsy), with all the OSCC from these patients scoring $0(n=5)$ or $1(n=1)$.

It has been reported that phosphorylated proteins are more labile, and that epitope degradation can occur within 30 minutes of ischaemia in formaldehyde (37) leading to loss of certain post-translational modifications (PTMs), specifically phosphorylation. Utilising the protein extraction protocol described in this study, the expression of PTMs has been shown to be consistently down-regulated across 4 different phosphorylation sites (ATR (s428), CHK1 (s317), FANCD2 (s331) and FANCG (s7)) in biopsy tissue destined to transform (T-OED) compared with that which was not (NT-OED). This finding is significant given the lack of availability of fresh tissue collections from cohorts of dysplasia patients and suggests that PTM biomarkers of transformation may be developed for FFPE tissue. This could potentially be used to influence treatment decisions in clinical practice or when utilised within the context of a clinical trial in the management of OED to stratify treatment/intervention arms.

The hypothesis that malignant change in OED involves alteration of the FA pathway is supported by both our immunohistochemistry and western blotting data. These reinforce the previously described differences observed in the appearance and site of OED in these two groups of patients(1) but are not simply a reflection of these differences as no associations were observed between site or appearance of lesion and PTM of these proteins. Samples from T OED lesions showed significant reduction in the phosphorylation of ATR, CHK1, FANCD2 and FANCG in comparison to non-transforming samples, indicating a lack of ATR-CHK1 activation following DNA damage and/or replicative stress. It may be argued that these observations are due to a lack of stimuli in the transforming group as they have a preponderance of non-smokers, but analysis of our data does not support this as no significant difference in PTM expression was observed between smokers and non-smokers. The ability of CHK1 to phosphorylate several functionally important sites for optimal function and activation of the FA pathway appears to be compromised in these patients which, it is proposed, will lead to the impairment of the functionality of the FA core complex and lead to a reduction in subsequent HRR activity $(20,25,29,38)$. In contrast, non-transforming samples showed high levels of FANCD2 s331 and FANCG s7 phosphorylation, 
indicating that these sites were successfully phosphorylated by activated $\mathrm{CHK} 1$, and could function effectively in DNA repair, thus reducing the burden of DNA damage in these cells and reducing the risk of malignant transformation.

FANCD2 monoubiquitylation, which is thought to be promoted by ATR-CHK1 mediated FANCD2 phosphorylation $(19,30)$, is a critical step in FA pathway activation $(29,39)$ and evidence suggests that a reduction in FANCD2 monoubiquitylation has a greater influence on genomic instability than down regulation of FANCD2 expression (40). In our study, it was observed that transforming samples have lower levels of FANCD2 monoubiquitylation (FANCD2 L expression) compared to non-transformers, and interestingly, they consistently displayed lower levels of total FANCD2 expression. These findings agree with our concurrent immunohistochemistry data where we observed a lack of FANCD2 protein expression in T-OED compared with NT-OED lesions.

The direct evaluation of the DNA sensing-signalling-damage repair cascade in OED has not been previously reported, although there is recent evidence that individuals with reduced, systemic, double strand break repair capacity are more prone develop to head and neck cancer (38). It has been suggested that activation of DNA damage response might be protective in the early stages of oral carcinogenesis, but progressive

deregulation over time could eventually result in the failure to suppress malignant transformation(41). The results of the current study indicate that additional evaluation of these pathways is worthwhile to understand their capability to predict malignant transformation in OED at the initial diagnostic biopsy, especially as time to transformation may be as long as 7 years $(1,3)$. Loss of heterozygosity (LOH) status at putative tumour suppressor gene loci (3p14, 9p21, 9p22 and 17p13) is currently the most reliable predictor in malignant transformation in OED (42-44) and there is evidence to suggest that LOH is secondary to homologous recombination deficiency/DNA damage repair deficiencies at 15 cancer sites, including head and neck squamous cell carcinoma (45), indicating a possible link with the current data.

In the process of validating the FANCD2-OED Risk score, a multi-centred setting with larger sample size would be desired, to control for as many variables as possible matched for smoking, clinical appearance, age and management (surveillance vs excision). This would with the numbers needed to treat analysis inform the sensitivity and specificity requirements of the potential for the FANCD2-OED Risk score to serve as a potential biomarker for malignant transformation in $\operatorname{OED}(46)$. This should explore possibilities of refining the processes presented in this study, to streamline the diagnostic pathway so that clinical application would be feasible and cost effective. It remains to be seen if the FANCD2-OED Risk score could be more accurate in the prediction of malignant transformation when compared to loss of heterozygosity(47), or perhaps they could be utilised jointly. This could inform the development of future novel treatment strategies in the management of OED.

\section{Conclusion}


This study has shown promise for the utility of the FANCD2-OED Risk score in evaluation of the status of the Fanconi Anaemia Pathway in OED and its impact on oral carcinogenesis. These findings require validation in a larger multi-centred cohort of patients. Future studies to further investigate the status of DNA damage repair pathways will improve the understanding of how this influences malignant transformation rates in OED and potentially identify personalised chemoprevention and/or therapeutic options.

\section{Declarations}

Ethical approval and consent to participate for publication : Ethical approval for this study was given by Sefton REC (now North West - Liverpool Central; reference number: EC 47.01). Consent was obtained for retrospective and prospective analysis of tissue samples and clinical records.

Availability of data and material : Anonymised data presented in this study will be made available on written request to the corresponding author for future systematic review and/or metanalysis

Competing interests : None declared

Funding :

- *Faculty of Dental Surgery (RCS England) Small Grant Award: 'Molecular and clinical determinants of malignant progression in oral dysplasia' (£10000)

- **British Association of Oral and Maxillofacial Surgeons Endowments Travel Expenses Grant $(£ 3000)$

- *British Association of Oral and Maxillofacial Surgeons/National Facial and Oral Research Centre (Saving Faces) Research Fellowship September 2013 - August 2014 (£8000)

- *Royal College of Surgeons England Honorary Research Fellowship 2012/13 - The molecular and clinical determinants of malignant progression in oral epithelial dysplasia $(£ 3000)$

*Funding provided for laboratory consumables costs

**Funding provided for travel associated with completion of laboratory work within the context of a parttime Doctorate of Medicine Thesis

Authors' contributions: MWSH, JBW, JMR and RJS were responsible for the study design. MWSH was the primary author of the article with contribution for the introduction from MPR and JG. MWSH and AT were primarily responsible for the immunohistochemistry components. JBW, MPR, JG, JMR and MWSH contributed to the protein extraction and Western blotting. All authors contributed to the review, edit and agreement of the final version of the submitted paper.

Acknowledgements: Preliminary findings of this paper has been presented as an oral abstract in the following meetings: European Congress of Head and Neck Oncology, Liverpool, 24-26 April 2014 and 
British Association of Oral and Maxillofacial Surgery Annual Scientific Meeting, Edinburgh, 2-4 July 2014. This article forms part of the Doctorate of Medicine thesis 'Clinical and Molecular Determinants of Malignant Transformation in Oral Epithelial Dysplasia' which has been deposited in the University of Liverpool Elements archive.

\section{Abbreviations}

NT - Non-transforming/stable oral epithelial dysplasia

OED - Oral epithelial dysplasia

OSCC - Oral squamous cell carcinoma

T - Malignant transforming oral epithelial dysplasia

\section{References}

1. Ho MW, Risk JM, Woolgar JA, Field EA, Field JK, Steele JC, et al. The clinical determinants of malignant transformation in oral epithelial dysplasia. Oral oncology. 2012;48(10):969-76.

2. Brennan M, Migliorati CA, Lockhart PB, Wray D, Al-Hashimi I, Axell T, et al. Management of oral epithelial dysplasia: a review. Oral surgery, oral medicine, oral pathology, oral radiology, and endodontics. 2007;103 Suppl:S19 e1-2.

3. Mehanna HM, Rattay T, Smith J, McConkey CC. Treatment and follow-up of oral dysplasia - a systematic review and meta-analysis. Head \& neck. 2009;31(12):1600-9.

4. Smith J, Rattay T, McConkey C, Helliwell T, Mehanna H. Biomarkers in dysplasia of the oral cavity: a systematic review. Oral oncology. 2009;45(8):647-53.

5. Nankivell P, Mehanna H. Oral dysplasia: biomarkers, treatment, and follow-up. Current oncology reports. 2011;13(2):145-52.

6. Macey R, Walsh T, Brocklehurst P, Kerr AR, Liu JL, Lingen MW, et al. Diagnostic tests for oral cancer and potentially malignant disorders in patients presenting with clinically evident lesions. Cochrane Database Syst Rev. 2015(5):CD010276.

7. Nankivell PC, Williams H, Bartlett JM, Mehanna H. Validation of tissue microarrays in oral epithelial dysplasia using a novel virtual-array technique. Journal of clinical pathology. 2012;65(12):1084-7.

8. Fan GK, Chen J, Ping F, Geng Y. Immunohistochemical analysis of P57(kip2), p53 and hsp60 expressions in premalignant and malignant oral tissues. Oral oncology. 2006;42(2):147-53.

9. Scott IS, Odell E, Chatrath P, Morris LS, Davies RJ, Vowler SL, et al. A minimally invasive immunocytochemical approach to early detection of oral squamous cell carcinoma and dysplasia. British journal of cancer. 2006;94(8):1170-5.

10. Antoniou AC, Beesley J, McGuffog L, Sinilnikova OM, Healey S, Neuhausen SL, et al. Common breast cancer susceptibility alleles and the risk of breast cancer for BRCA1 and BRCA2 mutation carriers: 
implications for risk prediction. Cancer research. 2010;70(23):9742-54.

11. Rudland PS, Platt-Higgins AM, Davies LM, de Silva Rudland S, Wilson JB, Aladwani A, et al. Significance of the Fanconi anemia FANCD2 protein in sporadic and metastatic human breast cancer. The American journal of pathology. 2010;176(6):2935-47.

12. Reed K, Ravikumar TS, Gifford RR, Grage TB. The association of Fanconi's anemia and squamous cell carcinoma. Cancer. 1983;52(5):926-8.

13. Deans AJ, West SC. DNA interstrand crosslink repair and cancer. Nature reviews Cancer. 2011;11(7):467-80.

14. Grompe M, D'Andrea A. Fanconi anemia and DNA repair. Human molecular genetics. 2001;10(20):2253-9.

15. D'Andrea AD, Grompe M. The Fanconi anaemia/BRCA pathway. Nature reviews Cancer. 2003;3(1):2334.

16. Moldovan GL, D'Andrea AD. How the fanconi anemia pathway guards the genome. Annual review of genetics. 2009;43:223-49.

17. Kupfer GM. Fanconi anemia: a signal transduction and DNA repair pathway. The Yale journal of biology and medicine. 2013;86(4):491-7.

18. Tomida J, Itaya A, Shigechi T, Unno J, Uchida E, Ikura M, et al. A novel interplay between the Fanconi anemia core complex and ATR-ATRIP kinase during DNA cross-link repair. Nucleic acids research. 2013;41(14):6930-41.

19. Andreassen PR, D'Andrea AD, Taniguchi T. ATR couples FANCD2 monoubiquitination to the DNAdamage response. Genes \& development. 2004;18(16):1958-63.

20. Qiao F, Mi J, Wilson JB, Zhi G, Bucheimer NR, Jones NJ, et al. Phosphorylation of fanconi anemia (FA) complementation group G protein, FANCG, at serine 7 is important for function of the FA pathway. The Journal of biological chemistry. 2004;279(44):46035-45.

21. Smogorzewska A, Matsuoka S, Vinciguerra P, McDonald ER, 3rd, Hurov KE, Luo J, et al. Identification of the FANCI protein, a monoubiquitinated FANCD2 paralog required for DNA repair. Cell. 2007;129(2):289-301.

22. Bunting SF, Nussenzweig A. Dangerous liaisons: Fanconi anemia and toxic nonhomologous end joining in DNA crosslink repair. Molecular cell. 2010;39(2):164-6.

23. Muller S. Update from the 4th Edition of the World Health Organization of Head and Neck Tumours: Tumours of the Oral Cavity and Mobile Tongue. Head and neck pathology. 2017;11(1):33-40.

24. Addis MF, Tanca A, Pagnozzi D, Crobu S, Fanciulli G, Cossu-Rocca P, et al. Generation of high-quality protein extracts from formalin-fixed, paraffin-embedded tissues. Proteomics. 2009;9(15):3815-23.

25. Zhi G, Wilson JB, Chen X, Krause DS, Xiao Y, Jones NJ, et al. Fanconi anemia complementation group FANCD2 protein serine 331 phosphorylation is important for fanconi anemia pathway function and BRCA2 interaction. Cancer research. 2009;69(22):8775-83. 
26. Wilson JB, Johnson MA, Stuckert AP, Trueman KL, May S, Bryant PE, et al. The Chinese hamster FANCG/XRCC9 mutant NM3 fails to express the monoubiquitinated form of the FANCD2 protein, is hypersensitive to a range of DNA damaging agents and exhibits a normal level of spontaneous sister chromatid exchange. Carcinogenesis. 2001;22(12):1939-46.

27. Lessard JL. Two monoclonal antibodies to actin: one muscle selective and one generally reactive. Cell Motil Cytoskeleton. 1988;10(3):349-62.

28. http://www.proteinatlas.org/ENSG00000175054-ATR/antibody.

29. Collins NB, Wilson JB, Bush T, Thomashevski A, Roberts KJ, Jones NJ, et al. ATR-dependent phosphorylation of FANCA on serine 1449 after DNA damage is important for FA pathway function. Blood. 2009;113(10):2181-90.

30. Pichierri P, Rosselli F. The DNA crosslink-induced S-phase checkpoint depends on ATR-CHK1 and ATR-NBS1-FANCD2 pathways. The EMBO journal. 2004;23(5):1178-87.

31. Geng L, Huntoon CJ, Karnitz LM. RAD18-mediated ubiquitination of PCNA activates the Fanconi anemia DNA repair network. The Journal of cell biology. 2010;191(2):249-57.

32. Wilson JB, Yamamoto K, Marriott AS, Hussain S, Sung P, Hoatlin ME, et al. FANCG promotes formation of a newly identified protein complex containing BRCA2, FANCD2 and XRCC3. Oncogene. 2008;27(26):3641-52.

33. Warnakulasuriya S, Reibel J, Bouquot J, Dabelsteen E. Oral epithelial dysplasia classification systems: predictive value, utility, weaknesses and scope for improvement. Journal of oral pathology \& medicine : official publication of the International Association of Oral Pathologists and the American Academy of Oral Pathology. 2008;37(3):127-33.

34. Nankivell P, Williams H, Matthews P, Suortamo S, Snead D, McConkey C, et al. The binary oral dysplasia grading system: validity testing and suggested improvement. Oral surgery, oral medicine, oral pathology and oral radiology. 2013;115(1):87-94.

35. Kujan O, Khattab A, Oliver RJ, Roberts SA, Thakker N, Sloan P. Why oral histopathology suffers interobserver variability on grading oral epithelial dysplasia: an attempt to understand the sources of variation. Oral oncology. 2007;43(3):224-31.

36. Guneri P, Epstein JB. Why are we still unable to accurately determine the malignant potential or the behavior of oral mucosal lesions? Oral oncology. 2017;71:177-9.

37. Vassilakopoulou M, Parisi F, Siddiqui S, England AM, Zarella ER, Anagnostou V, et al. Preanalytical variables and phosphoepitope expression in FFPE tissue: quantitative epitope assessment after variable cold ischemic time. Laboratory investigation; a journal of technical methods and pathology. 2015;95(3):334-41.

38. Joo W, Xu G, Persky NS, Smogorzewska A, Rudge DG, Buzovetsky O, et al. Structure of the FANClFANCD2 complex: insights into the Fanconi anemia DNA repair pathway. Science. 2011;333(6040):312-6.

39. Moldovan GL, D'Andrea AD. FANCD2 hurdles the DNA interstrand crosslink. Cell. 2009;139(7):1222-4. 
40. Gravells P, Hoh L, Solovieva S, Patil A, Dudziec E, Rennie IG, et al. Reduced FANCD2 influences spontaneous SCE and RAD51 foci formation in uveal melanoma and Fanconi anaemia. Oncogene. 2013;32(46):5338-46.

41. Nikitakis NG, Rassidakis GZ, Tasoulas J, Gkouveris I, Kamperos G, Daskalopoulos A, et al. Alterations in the expression of DNA damage response-related molecules in potentially preneoplastic oral epithelial lesions. Oral surgery, oral medicine, oral pathology and oral radiology. 2018;125(6):637-49.

42. Tsui IF, Rosin MP, Zhang L, Ng RT, Lam WL. Multiple aberrations of chromosome 3p detected in oral premalignant lesions. Cancer prevention research. 2008;1(6):424-9.

43. Shumway BS, Kresty LA, Larsen PE, Zwick JC, Lu B, Fields HW, et al. Effects of a topically applied bioadhesive berry gel on loss of heterozygosity indices in premalignant oral lesions. Clinical cancer research : an official journal of the American Association for Cancer Research. 2008;14(8):2421-30.

44. Zhang L, Poh CF, Williams M, Laronde DM, Berean K, Gardner PJ, et al. Loss of heterozygosity (LOH) profiles-validated risk predictors for progression to oral cancer. Cancer prevention research. 2012;5(9):1081-9.

45. Marquard AM, Eklund AC, Joshi T, Krzystanek M, Favero F, Wang ZC, et al. Pan-cancer analysis of genomic scar signatures associated with homologous recombination deficiency suggests novel indications for existing cancer drugs. Biomark Res. 2015;3:9.

46. Day RS. Planning clinically relevant biomarker validation studies using the "number needed to treat" concept. J Transl Med. 2016;14(1):117.

47. Cromwell I, Regier DA, Peacock SJ, Poh CF. Cost-Effectiveness Analysis of Using Loss of Heterozygosity to Manage Premalignant Oral Dysplasia in British Columbia, Canada. Oncologist. 2016;21(9):1099-106.

\section{Tables}

\begin{tabular}{|c|c|c|c|}
\hline \multicolumn{2}{|c|}{ Feature } & \multicolumn{2}{|c|}{ Binary score } \\
\hline & & 0 & 1 \\
\hline Nuclear & Extent & Absent-Focal & Multifocal-Widespread \\
\hline Staining & Intensity & Absent-Weak & Moderate- Strong \\
\hline Cytoplasmic & Extent & Absent-Focal & Multifocal-Widespread \\
\hline Staining & Intensity & Absent-Weak & Moderate-Strong \\
\hline
\end{tabular}

Table $1 \quad$ FANCD2 immunohistochemistry scoring system 


\begin{tabular}{|c|c|c|c|c|c|}
\hline Antibody against & Raised in & Company & Catalogue number & Dilution & Citations \\
\hline$\beta$-actin (C4) & Mouse & Santa Cruz & sc-47778 & $1: 5,000$ & $(27)$ \\
\hline ATR & Rabbit & Atlas & HPA028264 & $1: 1,000$ & (28) \\
\hline pATR & Mouse & \multicolumn{2}{|c|}{ Gift of Dr G. M. Kupfer } & $1: 1,000$ & $(25,29)$ \\
\hline CHK1 & Mouse & Santa Cruz & sc-56291 & $1: 1,000$ & $(30)$ \\
\hline pCHK1 (S317) & Rabbit & Calbiochem & DR1025 & $1: 1,000$ & (30) \\
\hline FANCD2* & Mouse & Santa Cruz & sc-28194 & $1: 500$ & (20) \\
\hline pFANCD2* $\left(\mathrm{S} 331^{\star}\right)$ & Mouse & \multicolumn{2}{|c|}{ Gift of Dr G. M. Kupfer } & $1: 1,000$ & $(25,29)$ \\
\hline FANCG* & Rabbit & Santa Cruz & sc-28219 & $1: 1,000$ & $(20)$ \\
\hline pFANCG* $\left(\mathrm{S}^{*}{ }^{\star}\right)$ & Mouse & \multicolumn{2}{|c|}{ Gift of Dr G. M. Kupfer } & $1: 1,000$ & $(29,31)$ \\
\hline
\end{tabular}

* selected as markers of activation of the Fanconi Anaemia pathway availability and reliability from previously published data $(25,32)$

Table $2 \quad$ Antibodies used in Western blot 


\begin{tabular}{|c|c|c|c|c|}
\hline & & $\begin{array}{l}\text { Non-transformers } \\
\qquad(\mathrm{n}=23)\end{array}$ & $\begin{array}{c}\text { Transformers } \\
(n=17)\end{array}$ & P value \\
\hline Age & Median (range) & $\begin{array}{c}55.9 \\
(27.9-78.4)\end{array}$ & $\begin{array}{c}57.5 \\
(37.5-85.2)\end{array}$ & 0.56 \\
\hline $\begin{array}{l}\text { Gender } \\
\text { n (\%) }\end{array}$ & $\begin{array}{l}\text { Male } \\
\text { Female }\end{array}$ & $\begin{array}{l}13(57) \\
10(43)\end{array}$ & $\begin{array}{c}6(35) \\
11(65)\end{array}$ & 0.22 \\
\hline $\begin{array}{l}\text { Site } \\
n(\%)\end{array}$ & $\begin{array}{l}\text { Lateral tongue } \\
\text { Floor mouth } \\
\text { Buccal } \\
\text { Alveolar mucosa }\end{array}$ & $\begin{array}{l}4(17) \\
11(49) \\
4(17) \\
4(17)\end{array}$ & $\begin{array}{c}10(58) \\
3(18) \\
4(24) \\
-\end{array}$ & 0.01 \\
\hline $\begin{array}{l}\text { Appearance } \\
n(\%)\end{array}$ & $\begin{array}{l}\text { Homogenous white } \\
\text { Red-white } \\
\text { Red }\end{array}$ & $\begin{array}{c}20(87) \\
2(9) \\
1(4) \\
\end{array}$ & $\begin{array}{c}7(41) \\
10(59) \\
- \\
\end{array}$ & 0.001 \\
\hline $\begin{array}{l}\text { Lesion size } \\
n(\%)\end{array}$ & $\begin{array}{l}<200 \mathrm{~mm}^{2} \\
\geq 200 \mathrm{~mm}^{2}\end{array}$ & $\begin{array}{l}5(22) \\
18(78)\end{array}$ & $\begin{array}{l}3(18) \\
14(82)\end{array}$ & 1 \\
\hline $\begin{array}{l}\text { Smoking } \\
\text { n (\%) }\end{array}$ & $\begin{array}{l}\text { Never } \\
\text { 5-20 pack years } \\
>20 \text { pack years }\end{array}$ & $\begin{array}{l}5(22) \\
7(30) \\
11(48)\end{array}$ & $\begin{array}{l}7(41) \\
8(47) \\
2(12)\end{array}$ & 0.05 \\
\hline $\begin{array}{l}\text { Alcohol } \\
\text { n (\%) }\end{array}$ & $\begin{array}{l}\text { Teetotal } \\
\text { Current drinker }\end{array}$ & $\begin{array}{l}6(26) \\
17(74)\end{array}$ & $\begin{array}{l}5(29) \\
12(71)\end{array}$ & 1 \\
\hline $\begin{array}{l}\text { Grade of dysplasia* } \\
\text { n (\%) }\end{array}$ & $\begin{array}{l}\text { Mild/moderate } \\
\text { Severe/Cis }\end{array}$ & $\begin{array}{l}20(87) \\
3(13)\end{array}$ & $\begin{array}{l}12(71) \\
5(29)\end{array}$ & 0.19 \\
\hline Definitive treatment & $\begin{array}{l}\text { Surveillance } \\
\text { Excision }\end{array}$ & $\begin{array}{l}17(74) \\
6(26)\end{array}$ & $\begin{array}{c}0 \\
17(100)\end{array}$ & 0.0001 \\
\hline
\end{tabular}

Table 3 Demographic and clinic-pathological features of patients $(n=40)(*$ at initial diagnostic biopsy) 


\begin{tabular}{|c|c|c|c|c|}
\hline \multirow[b]{2}{*}{$\begin{array}{l}\text { Site of } \\
\text { staining }\end{array}$} & \multirow[b]{2}{*}{ Pattern of staining } & \multicolumn{3}{|c|}{ Biopsy } \\
\hline & & $\begin{array}{c}\text { NT - first } \\
\text { diagnostic } \\
n(\%)\end{array}$ & $\begin{array}{c}T-\text { first } \\
\text { diagnostic } \\
n(\%)\end{array}$ & $\begin{array}{c}\mathrm{T}-\text { OSCC } \\
\text { diagnostic } \\
\mathrm{n}(\%)^{*}\end{array}$ \\
\hline & $\begin{array}{l}\text { Distribution } \\
\text { Absent/Focal } \\
\text { Multifocal/Widespread } \\
\text { Intensity** } \\
\text { Absent/Weak } \\
\text { Moderate/Strong }\end{array}$ & $\begin{array}{l}6(26) \\
17(74) \\
\\
13(57) \\
10(43) \\
\end{array}$ & $\begin{array}{c}4(24) \\
13(76) \\
\\
16(94) \\
1(6)\end{array}$ & $\begin{array}{c}9(60) \\
6(40) \\
\\
14(93) \\
1(7)\end{array}$ \\
\hline Cytoplasmic & $\begin{array}{l}\text { Distribution } \\
\text { Absent/Focal } \\
\text { Multifocal/Widespread } \\
\text { Intensity*** } \\
\text { Absent/Weak } \\
\text { Moderate/Strong }\end{array}$ & $\begin{array}{c}1(4) \\
22(96) \\
\\
5(22) \\
18(78)\end{array}$ & $\begin{array}{c}1(6) \\
16(94) \\
\\
8(47) \\
9(53)\end{array}$ & $\begin{array}{c}4(27) \\
11(73) \\
\\
10(67) \\
5(33)\end{array}$ \\
\hline
\end{tabular}

*Specimens for two patients were not retrievable from archival records

$\star \star$ NT v T (first diagnostic biopsy) P<0.008 (Mann-Whitney Test)

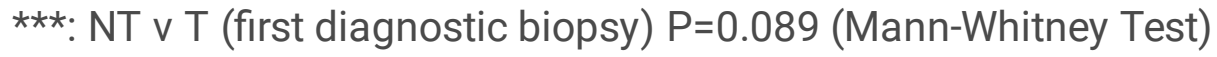

Table 4 FANCD2 immunohistochemistry scores in first diagnostic biopsy of non-transforming (NT) and transforming $(T)$ OED, together with paired OSCC for the transforming group

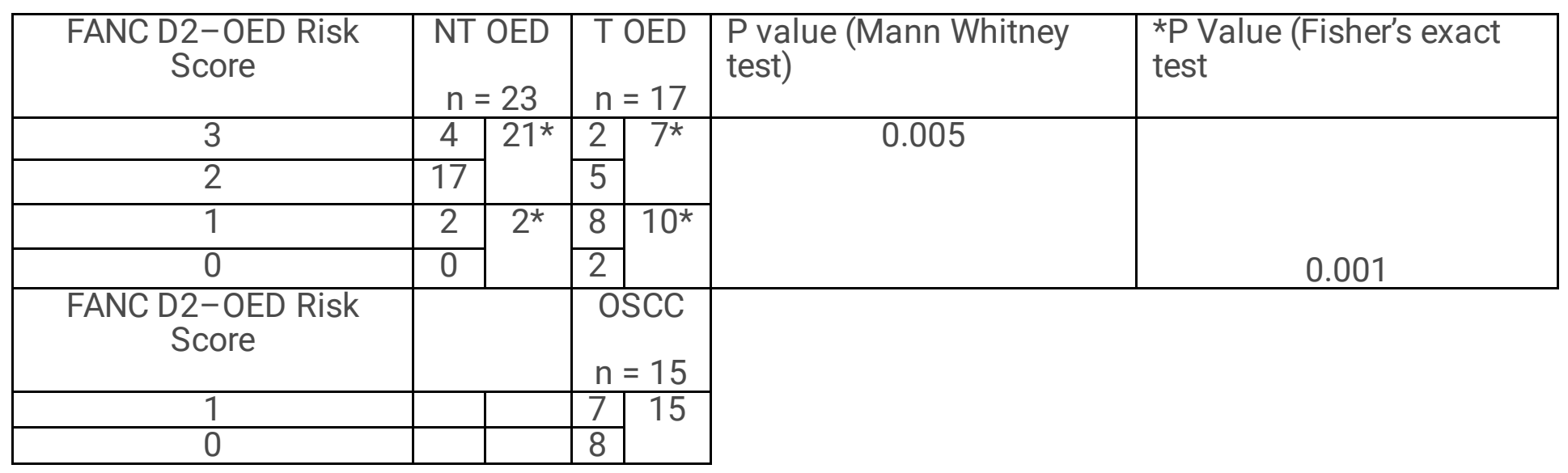

Illustration of how FANCD2-OED Risk Score was derived:

e.g. Severe dysplasia (0) with absent/weak nuclear staining (0) and moderate/strong cytoplasmic stain

(1) $=0+0+1=1$

Table 5 FANCD2 IHC-OED grade combined scores in stable OED (NT), OED which underwent malignant transformation $(T)$ and oral squamous cell carcinoma which had arisen from OED. 


\begin{tabular}{|c|c|c|c|c|c|}
\hline \multicolumn{2}{|c|}{$\begin{array}{l}\text { Normalised densitometry } \\
\text { score ratio* }\end{array}$} & $\begin{array}{c}\text { Low } \\
\text { expression } \\
\mathrm{n}(\%) \\
\end{array}$ & $\begin{array}{c}\text { Moderate } \\
\text { expression } \\
\mathrm{n}(\%) \\
\end{array}$ & $\begin{array}{c}\text { High } \\
\text { expression } \\
\mathrm{n}(\%) \\
\end{array}$ & $\begin{array}{l}\text { P value } \\
\text { (Fisher-Freeman-Halton } \\
\text { exact test) }\end{array}$ \\
\hline ATR & $\begin{array}{l}\mathrm{T} \\
\mathrm{NT}\end{array}$ & $\begin{array}{l}9 \\
7\end{array}$ & $\begin{array}{l}3 \\
8\end{array}$ & $\begin{array}{l}5 \\
8\end{array}$ & 0.24 \\
\hline pATR & $\begin{array}{l}\mathrm{T} \\
\mathrm{NT} \\
\end{array}$ & $\begin{array}{c}14 \\
1 \\
\end{array}$ & $\begin{array}{l}3 \\
9 \\
\end{array}$ & $\begin{array}{r}0 \\
13 \\
\end{array}$ & $<0.0005$ \\
\hline Chk1 & $\begin{array}{l}\mathrm{T} \\
\mathrm{NT}\end{array}$ & $\begin{array}{c}5 \\
10 \\
\end{array}$ & $\begin{array}{l}7 \\
5\end{array}$ & $\begin{array}{l}5 \\
8\end{array}$ & $>0.29$ \\
\hline pChk1 & $\begin{array}{l}\mathrm{T} \\
\mathrm{NT}\end{array}$ & $\begin{array}{c}13 \\
3\end{array}$ & $\begin{array}{l}3 \\
8\end{array}$ & $\begin{array}{c}1 \\
12\end{array}$ & $<0.0005$ \\
\hline FANCD2 (S \& L $\left.L^{\#}\right)$ & $\begin{array}{l}\mathrm{T} \\
\mathrm{NT}\end{array}$ & $\begin{array}{l}10 \\
10\end{array}$ & $\begin{array}{l}2 \\
8\end{array}$ & $\begin{array}{l}5 \\
5\end{array}$ & $<0.025$ \\
\hline pFANCD2-s331 & $\begin{array}{l}\mathrm{T} \\
\mathrm{NT}\end{array}$ & $\begin{array}{c}16 \\
1\end{array}$ & $\begin{array}{c}1 \\
12 \\
\end{array}$ & $\begin{array}{c}0 \\
10 \\
\end{array}$ & $<0.0005$ \\
\hline FANCG & $\begin{array}{l}\mathrm{T} \\
\mathrm{NT}\end{array}$ & $\begin{array}{c}4 \\
10\end{array}$ & $\begin{array}{l}6 \\
4\end{array}$ & $\begin{array}{l}7 \\
9\end{array}$ & 0.61 \\
\hline pFANCG-s7 & $\begin{array}{l}\mathrm{T} \\
\mathrm{NT}\end{array}$ & $\begin{array}{c}16 \\
1\end{array}$ & $\begin{array}{c}1 \\
10\end{array}$ & $\begin{array}{c}0 \\
12\end{array}$ & $<0.0005$ \\
\hline
\end{tabular}

- See materials and methods

\# Sum of 'small' and 'large' FANCD2 isoforms - L corresponds to the monoubiquitylated (activated) isoform p: phosphorylated proteins; 3331 \& s7: location of phosphorylation

Table $6 \quad$ Normalised protein expression in initial diagnostic biopsy of NT OED and T OED lesions

Figures 

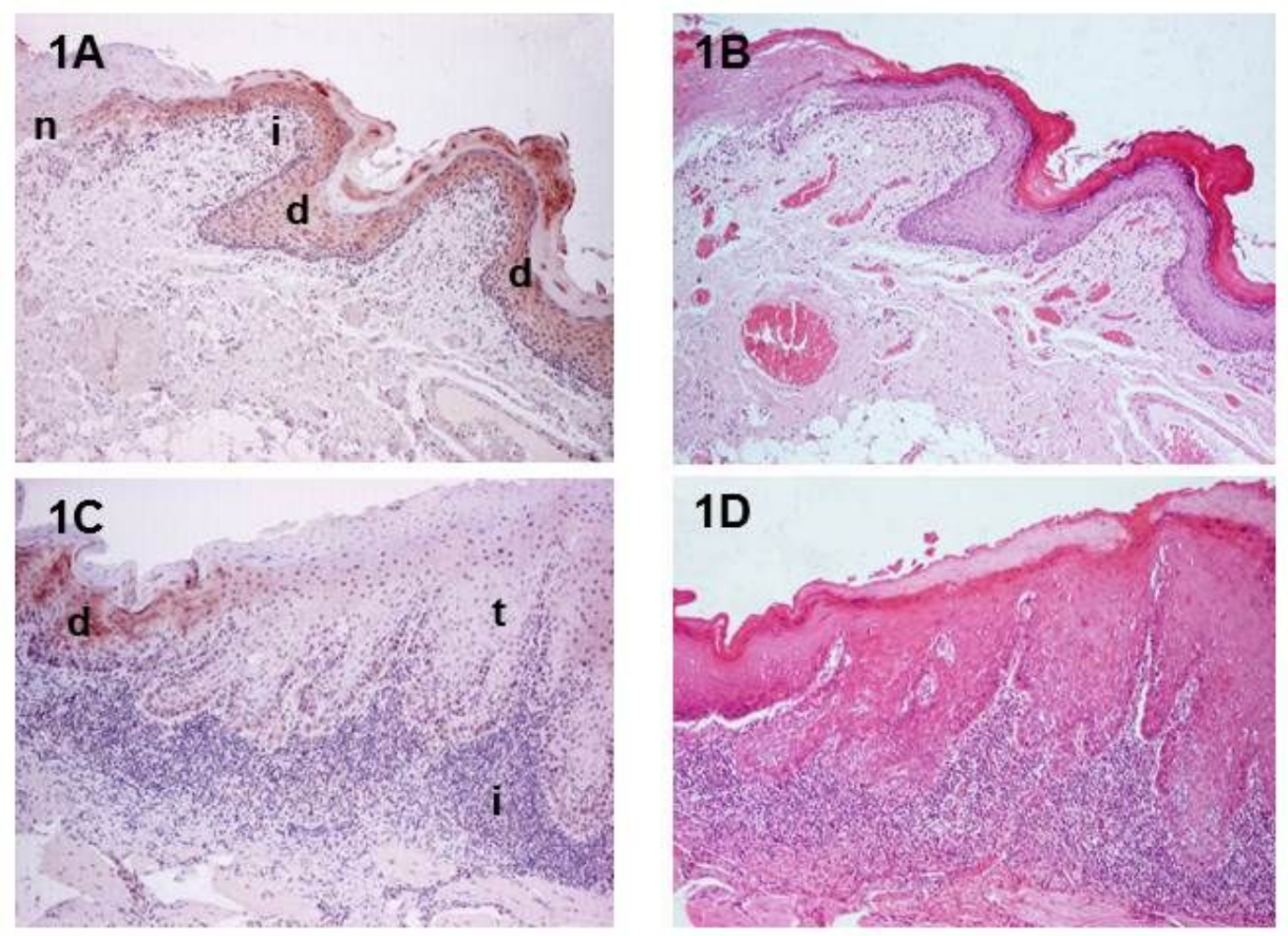

\section{Figure 1}

Immunohistochemistry for FANCD2. The photomicrographs illustrate the transition between normal (nondysplastic; $n$ ) and dysplastic (d) epithelium (A \& B); and dysplastic (d) epithelium and early squamous cell carcinoma ( $t$ ) ( $C \& D$ ). Differences in expression of FANCD2 between normal, dysplastic and invasive epithelia are seen: while variably strong, nuclear and cytoplasmic immunostaining is centred on the suprabasal layers of dysplastic epithelia, it is absent from normal epithelium ( $n$ in $1 \mathrm{~A}$ ) and lost from the 
tumour ( $\mathrm{t}$ in $\mathrm{C})$. Basal keratinocytes of the dysplastic epithelia are increased in numbers $(\mathrm{A})$ or show perturbed stratification and nuclear hyperchromatism (B). Inflammatory reaction (i) of variable density is seen underneath dysplastic epithelia and tumour (A \& C). B \& D are from adjacent sections stained with haematoxylin and eosin to allow comparison. Objective magnification $\times 4$.
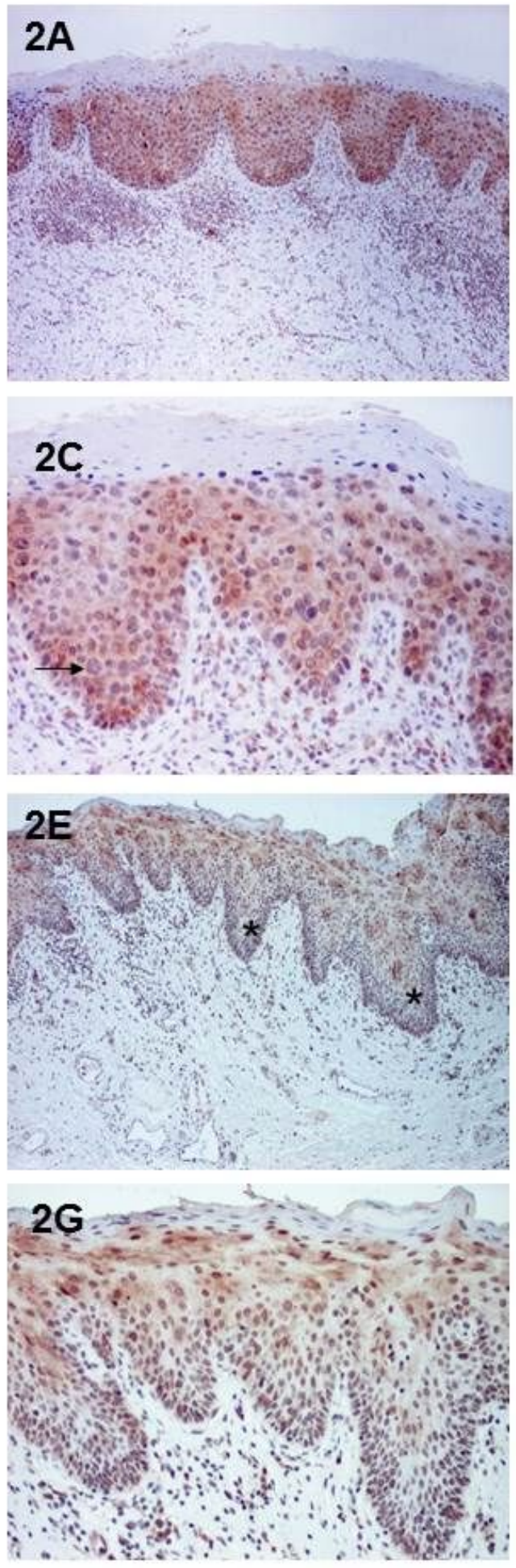
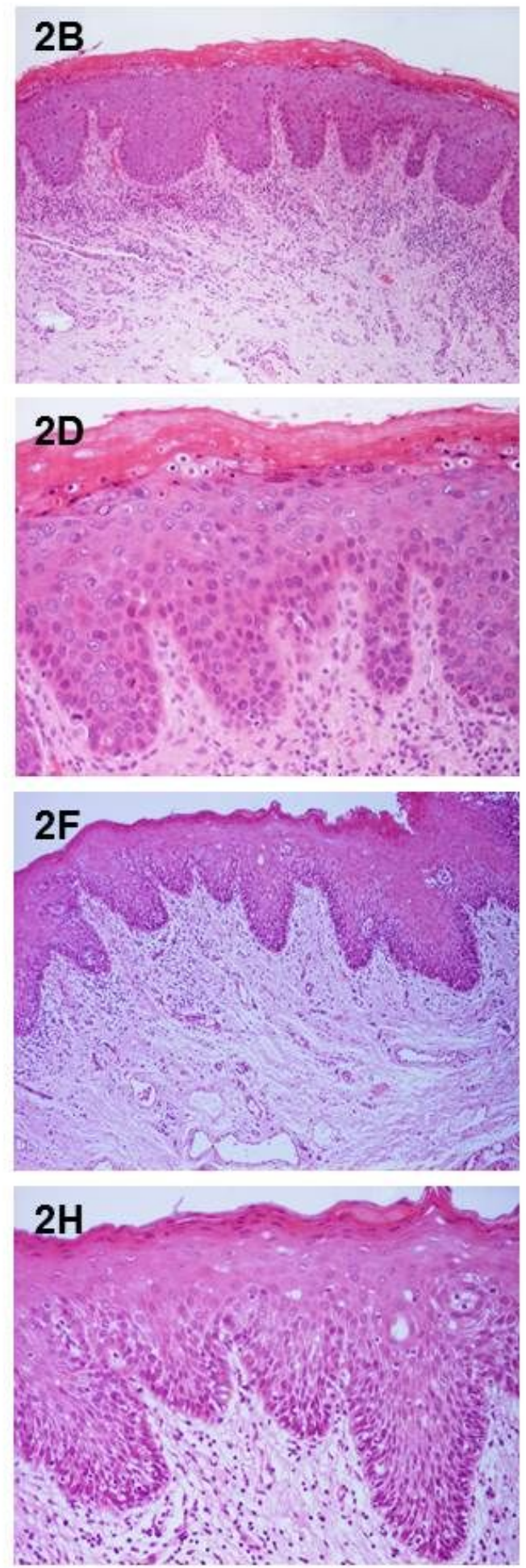

Figure 2 
Immunohistochemistry for FANCD2. The photomicrographs illustrate differences in expression of FANCD2 between NT OED (A, B, C, D) and T OED $(E, F, G, H)$. Although variably strong immunostaining decorates non-keratinising layers in NT OED (A \& C), it is absent from basal and parabasal, epithelial components (asterisks) in T OED (E \& G). Higher magnification allows appreciation of the differences and dysplastic features ( $C \& G$ ), including swollen, drop-shaped rete, perturbed stratification, enlarged nuclei (arrow), increased nuclear: cytoplasmic ratio (C), and markedly irregular lower epithelial border and increased numbers of cells having a basaloid phenotype $(G)$. Note the variable inflammatory reaction. $B$, $D, F \& H$ are from adjacent sections stained with haematoxylin and eosin to allow comparison. Objective magnification $\times 4(A, B, E, F) ; \times 10(C, D, G, H)$.
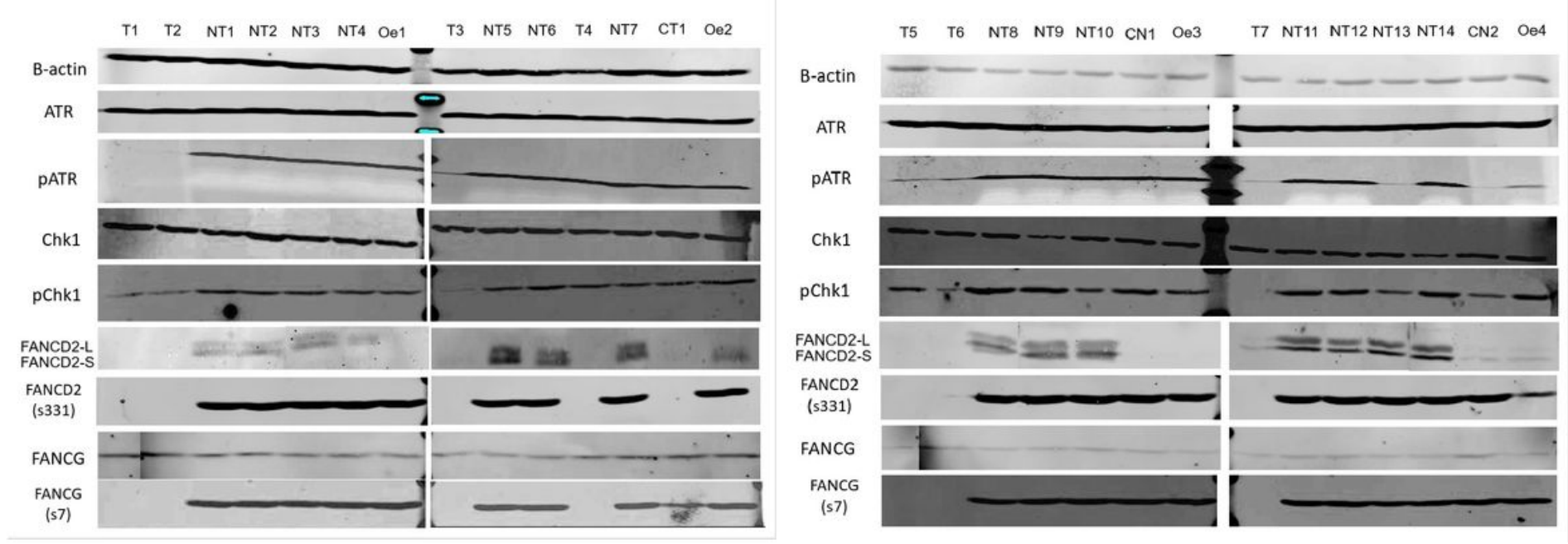

Figure 3

Representative example of Western blot expression analysis of DNA damage sensing and repair proteins. NT: first diagnostic biopsy of a non-transforming OED; T: first diagnostic biopsy of a transforming OED; Oe: normal oesophagus; $\mathrm{CT}$ : control OSCC from a different cohort; $\mathrm{CN}$ : control (morphologically) normal from margin of OSCC resection. 